\title{
Liver injuries- a tertiary rural medical college hospital experience
}

\author{
Prabhu S.G. ${ }^{1}$, Abraham G. ${ }^{2}$, Jayant B. N. ${ }^{3}$ \\ ${ }^{1}$ Dr. Satish G. Prabhu, Professor and Unit Chief, ${ }^{2}$ Dr. George Abraham, Assistant Professor, ${ }^{3}$ Dr. Jayant B.N. Assistant \\ Professor, All authors are affiliated to the Department of General Surgery, MOSC Medical College, Kolenchery, Kochi, \\ Kerala 682311, India.
}

Corresponding Author: Dr. George Abraham, Assistant Professor, Department of General Surgery, MOSC Medical College, Kolenchery, Kochi, Kerala 682311, India. Email: drgeorgeabr@gmail.com

\begin{abstract}
Background: Liver is the most frequently injured solid intra abdominal organ in abdominal trauma. Exsanguination is the main cause of death due to liver trauma. Although non-operative management of hepatic trauma has been utilized with increasing frequency a significant percentage of unstable liver trauma still require operative treatment. The aim of the present study was to examine the results of the operative treatment of patients with unstable liver trauma and prevent prolonged hospital stay. Patients admitted following Liver Trauma in the department of General Surgery, MOSC medical college Hospital, Kolenchery duringthe two year period from January 2013 to January 2015 were included in the study. Methods: This was a retrospective study of patients with Hepatic trauma admitted to the department of General Surgery, MOSC Medical College Hospital, Kolenchery, Ernakulam from January 2013 to January 2015. The diagnosis of Liver trauma was made pre-operatively with imageology and assessed clinically prior to surgery. Severity of liver injury was graded, treatment details collected and factors contribution to prolonged hospital stay were noted. These patients were later followed up for a period of one year. Results: During the two year period, 105 patients who were diagnosed to have liver injury were included in the study. Age varied from $12-75$ years. Males outnumbered females ( $88.5 \%$ vs. $11.43 \%$ ). 93 patients were with blunt abdominal trauma (88.5\%). 66(62.85\%) patients were in shock when they presented to the ER. 36 (37.15\%) patients were haemo- dynamically stable. 57 patients (54.28\%) had associated injuries like multiple rib fractures and splenic injuries. Exploratory laparotomy and control of bleeding, hepatorapphy and local debridement was done. Nine patientsrequired relaparotomy and omental packing. Fifteen patients succumbed to liver injury. The postoperative period was delayed in those patients who had other visceral injury. Follow up of cases for a period of 1 year was done and there was no late complication like intra-abdominal abscess, coagulopathy, bile leak or hepatic abscess. Conclusion: Emergency laparotomy with hemostasis and repair liver injury in unstable cases and select stable cases savestime and life of the patient, it is cost effective and hospital stay and systemic complications are minimal
\end{abstract}

Key words: Liver injury, Laparotomy, Hemostasis, Liver injury repair

\section{Introduction}

The Liver is the most commonly injured intraabdominal solid organ in both blunt and penetrating trauma because of its size and location [1]. Exsanguination is the major cause of death in hepatic trauma with a (mortality [S1] of 10-15[2]. Liver trauma should be considered in all patients with penetrating or blunt trauma, particularly in hypotensive patients with penetrating or blunt trauma on the right side [3]. Blunt trauma more commonly affects the right lobe of liver, particularly the posterior sector [3]. Although conservative treatment of low grade liver injuries is practiced

Manuscript Received: $10^{\text {th }}$ September 2018

Reviewed: $20^{\text {th }}$ September 2018

Author Corrected: $24^{\text {th }}$ September 2018

Accepted for Publication: $28^{\text {th }}$ September 2018 nowadays emergency laparotomy and hemostasis with repair of liver injury has a definite role in unstable cases and reduces the mortality and morbidity to a great extent [4]. The criteria for conservative treatment of liver injuries includes Hemodynamic stability, normal mental status and absence of clear indications for laparotomy such as peritoneal signs, low grade injuries (grade1 to 3) and transfusion requirements less than 2 units of blood [5].

Most series report a success rate of almost $90 \%$ for conservative treatment of liver injuries. The success rate is $95 \%$ for low grade liver injuries 1 to 3 which falls to $75 \%$ for grade $1 \mathrm{~V}$ to VI injuries [6]. Conservative 


\section{Original Research Article}

management has shown a lower rate of complication (0-11\% [6]. However a significant number of liver trauma cases especially the grade 3 to grade 5 cases benefit by timely and early laparotomy, hemostasis and repair of liver injury especially in a rural hospital where prolonged critical care is not always possible.

Angiography and selective embolization is increasingly used in the treatment of persistent bleeding cases and this may result in more cases being treated conservatively [5]. However these modalities may not always be available.

The principles of operative treatment are the same for all cases of liver injuries. They include damage control surgery that includes arrest of bleeding, removal of devitalized tissueand prevention of biliary complications in unstable patients [7].

Most of the liver injuries can be managed with simple procedures like suturing, debridementor packing with omentum gel foam. The mortality and morbidity associated with liver injuries varies drastically from $1.5 \%$ to $31 \%$.

Mortality is low with penetrating injuries whereas the mortality associated with blunt trauma is as high as $31 \%$ depending on the mechanism of injury. Early surgical intervention reduces mortality and morbidity and saves patients life, time and money.

\section{Material and Methods}

Study Setting: The study was conducted in department of General Surgery MOSC Medical College, Kolenchery.

Study Design: Retrospective observational study

Study Period: Between January 2013 and February 2015.

Sample Size: 105 Cases of blunt or penetrating abdominal injury with preoperative ultra sound or CT scan diagnosis of liver injury
Exclusion:- We did not include patients who died at the scene or on their way to hospital. Patients who arrived in the Emergency department in a state of cardiorespiratory arrest and whose period of attempted resuscitation did not exceed 15 minutes were also excluded from the study.

Ethical Consideration: The study was approved by the institutional human ethics committee. Informed written consent was obtained from all the study participants.

Study procedure- Patients fulfilling the inclusion criteria (105 cases) were identified and studied who presented to our emergency department with liver injury. Patients were resuscitated in the Emergency department and optimized.

Diagnosis was established by either by surgery, organ imaging by computerized tomography or ultrasound.

Based on the imaging and clinical findings that included vital signs patients were divided into stable and unstable cases and classified (according [S1] to the liver injury scale (1994 revision of the American Association for the Surgery of Traumaliver injury scale), the most widely used liver injury grading system used at the time of study [4].

Unstable cases were taken up for laparotomy where as the stable cases were initially treated conservatively and those cases which showed clinical deterioration during the follow up were taken up surgery.

Emergency exploratory laparotomy was done through a midline vertical incision and hemostasis was attempted with Pringles maneuver, Gauze packing, simple ligation, hepatorapphy with catgut and with omental packing and debridementin those cases where there was uncontroll able hemorrhage.

Postoperative follow up was like in any other case of emergency laparotomy but with specific emphasis on vital signs, clinical improvement or deterioration of the patient.

\section{Results}

During the study period 105 patients who presented to emergency department with liver injury were (90 males [85.71\%] and 15 females [14.29\%]). The majority of patients $93(88.57 \%)$ sustained blunt trauma and 12 had penetrating injury $(11.43 \%)$. Patients belonged to the wide age group from 12 years to 71 years.

Maximum incidence was in the 21-30 age group (28.7\%) followed by 10-20 age group (25.71\%). 72(68.57\%) patients were brought to the ER following road traffic accident, 21(20\%) patients were admitted with a history of fall from height. and $12(11.4 \%)$ patients were admitted following stab injury. 
Original Research Article

Table-1: Grades of Injury \& condition of patients.

\begin{tabular}{|c|c|c|}
\hline Grade of liver injury & Stable & Unstable \\
\hline i & 9 & 6 \\
\hline ii & 15 & 39 \\
\hline iii & 15 & 12 \\
\hline iv & 0 & 6 \\
\hline v & 0 & 3 \\
\hline vi & 0 & 0 \\
\hline Total & $\mathbf{3 9}$ & $\mathbf{6 6}$ \\
\hline
\end{tabular}

According to table 1 , in our study majority of the cases were unstable cases $66 / 105(62.8 \%)$. The majority of the cases were grade 2 liver injury 54/105(51.4\%). Unstable cases with grade 2 liver injury accounted for 39/68 (57.3\%) cases.

Associated Injuries- The associated injuries were as follows.

- Patients with multiple rib fractures- 24 patients (22.85\%)

- Haemothorax-18 patients (17.42\%)

- splenic injury- 3 patients

- multiple mesenteric tear-12 patients

- head injury 10 patients

- long bone fracture 16 patients

Among these multiple rib fractures, head injury and long bone fractures influenced the post-operative recovery and were significant determinants of successful recovery. However the major determinants were hemorrhage and shock.

Surgery- All 69 unstable patients including three patients in grade 2 liver injury who were initially stable at presentation were optimized prior to exploratory laparotomy. Abdomen was entered through midline vertical incision. Hemorrhage was controlled by digital pressure, suture ligation, diathermy, omental packingand lobectomy depending on the intraoperative findings. Re-laparotomy was done in 9 patients.Rebleeding in three patient which was controlled by catgut suturing, omental packing in one patient and Segmentectomy was done in the other two. Three patients were operated for perihepatic packing removal done as part of damage control surgery and the rest three patient required relaparotomy for drainage of hematoma and perihepatic collection since radiological intervention was successful. During the postoperative period fifteen patients deteriorated and they couldnot be revived in spite of resuscitation. The cause of death in one patient was Grade v liver injury with massive bleed and cardiac arrest during the immediate postoperative period. The second patient had Grade 3 liver injury with splenic injury and diaphragmatic hernia who died on the 5th postoperative period following pneumonia and sepsis. Three patients died because of massive rebleed during the immediate postoperative period. Four patients died due to associated head injury. Five patients due to sepsis and renal dysfunction. And one patient developed myocardial infarction in post op period. The mortality rate in our study was $14.28 \%$. Various studies have reported mortality rates ranging from $18 \%$ to $36 \%$.

Fifteen patients developed sepsis out of which ten survived. Wound infection was noted in twenty-three patients. Blood transfusion was a major concern and 2 to 50 units of blood was transfused. FFP and platelets were transfused during the postoperative period. Transfusion requirements were judged by perioperative blood loss, serial blood investigations and clinical judgment. Patients other than those with associated injuries like head injury, long bone fracture had earlier discharge. The mortality rate in our study was $14.70 \%$ which was much less than the rate reported in literature. The unstable cases were taken up for laparotomy and were treated by suture ligation, digital pressure, diathermy coagulation, omental packing and debridement. All the procedures were equally good. The survival rate was (85.7\%) with (early [S1] recovery, short hospital stay, and minimal complications. In our study we found that early operative intervention in unstable cases in spite of being low grade injuries results in early recovery with very few complications. Regular postoperative follow up was done for a period of 1 year and all the patients were asymptomatic as proved by the laboratory and imageology investigations. 


\section{Original Research Article}

\section{Discussion}

Liver is the commonest intraabdominal solid organ to be injured in blunt orpenetrating trauma because of its size, location and relative fragile parenchyma [1]. Severe hepatic trauma is a major cause of death in abdominal trauma. With the developments in imageology most hepatic injuries can be treated conservatively. The criteria for non operative treatment of hepatic injuries includes hemodynamic stability, absence of peritonitis, low grade hepatic injuries and transfusion requirements of less than 2 units of blood [5]. Most series show a success rate of $90 \%$ for conservative treatment [8].

However a small but significant percentage of unstable patients with liver injury benefit from early and timely surgical intervention resulting in improved quality of life to the patient. Most hepatic injuries were caused by blunt trauma occurring during motor vehicle accidents which is as per literature [9]. Most hepatic injuries were associated with other visceral injuries 60/105 (57.1\%) as reported other literature [10]. Mortality in low grade hepatic injuries (grade 1 to 111) is almost always caused by associated injuries and not by liver injury as describes in literature $(10.4 \%)$. In our study the mortality in low grade liver injuries was 12/96 (12.5\%).

However mortality associated with high grade injuries (grades IV to VI) varies from $18 \%$ to $36 \%(11)$. In our study the mortality in high grade liver injury was $3 / 9$ (33.3\%). Presence of shock at the time of admission is associated with higher mortality [11]. The aim of treatment while dealingwith an unstable liver injury was to control bleeding as quickly as possible and thus limit the extent of liver injury. Direct suturing of the bleeding artery,suture approximation of the liver wound edge (hepatorapphy), hepatic artery ligation, omental packing, resectional debridement, anatomic hepatic resection, perihepatic packing are the procedures commonly employed [12]. In our study majority of the bleeding was controlled with simple suturing and diathermy coagulation.

When active bleeding is encountered on table inflow occlusion of the liver should be performed by compressing the hepato- duodenal ligament with a vascular clamp (Pringles maneuver). When bleeding continues it is from the hepatic veins or the IVC[13]. Direct suturing of the arteries isrecommended when the bleeding is from the branches of the hepatic arteries or tributaries of the portal veins [12]. When a segment of the liver is damaged, debridement of the devitalized liver tissue with concomitant suture ligation of the bleeding vessels is done [14] [15]. Omental packing deep in the liver with reinforcing sutures is a useful procedure. Omentum provides an excellent source of macrophages and it fills a potential dead space with viable tissue [16]. In our study we had done omental packing in one patient. Anatomical resection of the liver is seldom done and it is replaced with resectional debridement. In a large series of 5000 cases of hepatic trauma hepatic resection was done only in $7.5 \%$ patients and the mortality were as high as $52 \%$ [17]. Perihepatic packing is done by keeping roller guaze around the liver and is very useful in patients with other intra-abdominal injuries and shock. Re exploration for pack removal was done after 48 to 72 hours. The association of hepatic abscess as per literature was $29 \%$ [18] [19]. However in our study there was no reported case of hepatic abscess development during the follow period. The most prevalent complication is wound infection. It was more commonly seen in those with poly trauma.

The cause of mortality in low grade injuries (1 to 3 ) is associated injuries while in high grade injuries (4 to 6) the liver injury itself resulting in exsanguinations the cause of death

\section{Conclusion}

Based on our findings and study we found that for unstable cases of liver injury irrespective of the grade of liver injury timely surgical intervention has a role in saving lives, reducing hospital stay and relatively uneventful post-operative period and follow up. It was found that emergency laparotomy and control of bleeding and repair of liver injury by simple methods was found to be very affective and improve the quality of the patient. Associated visceral injuries contributed to the mortality and morbidity of the patient. According to the literature althoughconservative treatment of liver injury is recommended for grade 1 , grade 2 and select grade3 caseswe found that early intervention irrespective of grade of injury in unstable cases improved the quality of life of patient.

Contribution by authors: Dr Satish G Prabhuguided the study and was instrumental in preparing the manuscript. Dr George Abraham edited the manuscript and added the requisite inputs as and when required, $\mathrm{Dr}$ Jayant B N compiled the cases and prepared the draft.

Acknowledgement- The Department of Radiology, M.O.S.C Medical, College 


\section{Original Research Article}

\section{Declaration}

Conflict of interest: None declared.

Funding: Nil, Permission from IRB: Yes

\section{References}

1. Ahmed N, Vernick JJ. Management of liver trauma in adults. J Emerg Trauma Shock. 2011;4(1):114-9.

2. Gourgiotis S, Vougas V, Germanos S, Dimopoulos $\mathrm{N}$, Bolanis I, Drakopoulos S, et al. Operative and nonoperative management of blunt hepatic trauma in adults: a single-center report. J Hepatobiliary Pancreat Surg. 14(4):387-91.

3. Jiang H, Wang J. Emergency strategies and trends in the management of liver trauma. Front Med. 2012 Sep;6 (3): 225-33.

4. Coughlin PA, Stringer MD, Lodge JPA, Pollard SG, Prasad KR, Toogood GJ. Management of blunt liver trauma in a tertiary referral centre. BJS. 91 (3): 317-21.

5. Stassen NA, Bhullar I, Cheng JD, Crandall M, Friese $\mathrm{R}$, Guillamondegui O, et al. Nonoperative management of blunt hepatic injury: An Eastern Association for the Surgery of Trauma practice management guideline. J Trauma Acute Care Surg. 2012 Nov;73:S288-93.

6. Asfar S, Khoursheed M, Al-Saleh M, Alfawaz AA, Farghaly MM, Nur AM, et al. Management of Liver Trauma in Kuwait. Med Princ Pract. 2014; 23 (2): 160-6.

7. Doklestic K, Djukic V, Ivancevic N, Gregoric P, Loncar Z, Stefanovic B, et al. Severe blunt hepatic trauma in polytrauma patient: Management and outcome. Srp Arh Celok Lek. 2015;143(7-8):416-22.

8. Parks RW, Chrysos E, Diamond T. Management of liver trauma. British journal of surgery. 1999 Sep 1;86 (9): 1121-35.
9. Stracieri LD da S, Scarpelini S. Hepatic injury. Acta Cir Bras. 2006; 21(suppl 1):85-8.

10. Trunkey DD, Shires GT, Mc Clelland R. Management of liver trauma in 811 consecutive patients. Ann Surg. 1974 May;179(5):722-8.

11. Vatanaprasan T. Operative Treatment of Hepatic Trauma in Vachira Phuket Hospital. 2005;88:11.

12. Feliciano DV. Continuing evolution in the approach to severe liver trauma. Annals of surgery. 1992 Nov; $216(5): 521$.

13.Badger SA, Barclay R, Campbell P, Mole DJ, Diamond T. Management of Liver Trauma. World J Surg. 2009 Dec;33(12):2522-37.

14. Cox EF, Flancbaum L, Dauterive AH, Paulson RL. Blunt trauma to the liver. Analysis of management and mortality in 323 consecutive patients. Annals of surgery. 1988 Feb;207(2):126.

15. Hollands MJ, Little JM. The role of hepatic resection in the management of blunt liver trauma. World journal of surgery. $1990 \mathrm{Jul}$ 1;14(4):478-82.

16. Stone HH, Lamb JM. Use of pedicled omentum as an autogenous pack for control of hemorrhage in major injuries of the liver. Surg Gynecol Obstet. 1975 Jul; 141 (1): $92-4$.

17. Pachter HL, Spencer FC. The management of complex hepatic trauma. Phila Saunders. 1983;241-249.

18. Feliciano DV, Mattox KL, Burch JM, Bitondo CG, Jordan JG. Packing for control of hepatic hemorrhage. J Trauma. 1986;26(8):738-743.

19. Reed 2nd RL, Merrell RC, Meyers WC, Fischer RP. Continuing evolution in the approach to severe liver trauma. Ann Surg. 1992;216(5):524.

\section{How to cite this article?}

Prabhu S.G, Abraham G, Jayant B. N. Liver injuries- a tertiary rural medical college hospital experience. Surgical Update: Int J surg Orthopedics.2018;4(3):121-125.doi:10.17511/ijoso.2018.i03.05. 